\title{
Lipid Changes in the Midgut of White Grub Adults Of Leucopholis lepidophora (BL)
}

\author{
S. B. More \\ Department of zoology, P. V. P. Mahavidyalaya, Kavathe Mahankal, Dist. Sangli, (M.S.) India. 416405
}

\begin{abstract}
The total lipids (TL), neutral lipid (NL) and phospholipids (PL) with their constituents were studied in midgut of white grub adults of Leucopholis lepidophora by employing thin layer chromatography (TLC) and bioassay technique. The midgut of male adults exhibits three fold more neutral lipids than the female adults midgut. The percentage of neutral lipids were 90.54 and 88.27, whereas NL:PL ratio were 10:1 and 7:1 respectively. Neutral lipids consisted of triacylglycerol (TG) as the major component, monoacylglycerol (MG) and diacylglycerol $(D G)$ in moderate concentration, whereas cholesterol (CHO), cholesterol ester (CE) and free fatty acids (FFA) in low concentration. Phosphatidyl-choline (PC) and phosphatidyl-ethanolamine (PE) were major phospholipid constituents. Phosphatidyl-inositol (PI), lysophosphatidyl- choline (LPC), sphingomyelin $(S P G)$ phosphatidyl-serine (PS), and phosphatidiec acid (PA) in low amound. The physiological significance with their constituents were discussed in relation with midgut.
\end{abstract}

Key Words: Neutral Lipids, Phospholipids, TLC, Midgut and Leucopholis lepidophora.

\section{Introduction}

Fats are important dietary constituents of most insects and it must be assumed that digestive lipases are important components of the midgut enzyme elements. However, conclusions concerning the nature of triacylglycerols hydrolysis in insect gut are confined to studied in Periplaneta americana. (Gilbert et al; 1965, Bollade et al; 1970; Hoffman and Downer, 1979, Male and Storey, 1981), L. migratoria and Polia adjuncta (Male and Storey, 1981). Cholesterol absorption in the cockroach P. americana was studied by Joshi and Agarwal (1976, 1977). They observed that, cholesterol abosroption in the alimentary canal takes place predominantly in the oesophagus and crop followed by the gizzard and gastric caeca. Further they observed that, in omnivorous insects cholesterol was absorbed in the foregut predominantly in the crop. In phytophagus insects and some others the midgut was the main site of cholesterol absorption.

The digestion and absorption of lipids in insects were studied by Turunen (1978). He reported that DG as the key intermeditary metabolites in glyceride translocation across the insects gut wall. Both triacylglycerol and phospholipids were hydrolysed in the intestinal lumen of the insects. The synthesis of DG acetyltransferase from crop, midgut and fat body tissue of P. americana was studied by Hoffman et al; (1979). They reported that the high rate of acylation was observed in the midgut, but the crop and fat body also displayed a capacity for acylation of MG. Similar findings were reported on T. infestans by Rimoldi et al; (1985).

\section{Materials And Methods}

The male and female adults of Leucopholis lepidophora were collected from sugarcane field of Sangrul village, 18 k.m. away from Kolhapur city. The midgut from male and female adults were dissected out and used for the lipid extraction.

\section{Extraction of Lipids}

The midgut of male and female adults were weighed accurately and homogenate with $20 \mathrm{ml}$. of chloroform-methanol $(2: 1, \mathrm{v} / \mathrm{v})$ at room temperature. The homogenate was allowed to stand for $2-3$ hours at $4^{0} \mathrm{C}$ and filtered. The filtrate was washed according to the method of Folch et al., (1957) and evaporated in vacuum at $40^{0} \mathrm{C}$. The lipid samples were weighed and preserved at $-20^{0} \mathrm{C}$ until further use. The total lipid in the sample was determined gravimetrically.

\section{Separation of Neutral Lipids and Phospholipids}

The neutral and phospholipids were separated by thin layer chromatography (TLC) using silica gel G. and about 200 mesh containing $\mathrm{CaSo}_{4}$ as a binder, (E Merck, Germany). The TLC plates (20 X $20 \mathrm{~cm}$.) were prepared according to Wagner et al., (1961). The known quantities of samples dissolved in chloroform were applied with Hamilton microsyringe, No. 8206-B on activated plates. For neutral lipids the plates were developed in hexane (b.p. $\left.65-70^{\circ} \mathrm{C}\right)$ diethyl ether-acetic acid $(85: 15: 2, \mathrm{v} / \mathrm{v})$ as recommended by Gloster and 
Flecter (1966). The phospholipid preparations were developed in chloroform-methanol-ammonia (115:45:5 v/v) as recommended by Barwal and Kalra (1988). The standards of neutral and phospholipids (Sigma, U.S.A.) were co-chromatographed in each respective run and then plates were kept in iodine chamber for charactrization of individual spots of lipids.

\section{Estimation of Neutral and Phospholipid}

The iodine was allowed to evaporate and the silica gel from the individual spots of glycerides was scraped and eluted in $1 \mathrm{ml}$ of diethyl-ether and assayed according to Viogue and Holman (1962). The cholesterol and its esters were estimated according to Abell et al., (1952). The rest of the neutral lipid components were assayed titrometrically by method of Skipski et al., (1967). The Phospholipid was determined by the method of Marinette (1967).

\section{Neutral Lipids}

\section{Results}

TLC of separation of various neutral lipid components are illustrated in Plate No.1 Fig. A whereas, Table 1 exhibits quantitative variations in the neutral lipid components.

The neutral lipids in midgut of male and female adults were measured 61.86 and $18.44 \mathrm{mg} / \mathrm{gm}$. wet weight of tissues respectively. The neutral lipids consisted of six components. The triacylglycerol being the major component both in male and female adults midgut. The percent value of triacylglycerol in midgut of male was 62.63 whereas in female midgut it was 88.27. The monocyglycerol, diacyglycerol and cholesterol were found in moderate concentration whereas, cholesterol ester and free fatty acids were occurred in trace amount.

\section{Phospholipids}

The phospholipids are illustrated in Fig. B and Table 2. The phospholipid in male and female midgut of L.lepidophora were measured 6.392 and $2.450 \mathrm{mg} / \mathrm{gm}$. wet weight of tissues respectively. The Thin layer choromatographic separation of phospholipids indicated the following constituents, PI, LPC, SPG, PS, PC, PE and PA. A critical study of Table 2 indicated that among the phospholipids PC and PE were the major constituents whereas PI, LPC, SPG, PS and PA in were in minor concentration. The percent value of PC and PE in midgut of male and female adults was 39.06, 37.6 and 49.02, 38.00 respectively.

\section{Discussion}

In insects the digestive tract is divided into three parts, the first part is called foregut, which is mainly used for storage of food. The second part is called midgut, which is mainly responsible for the digestion and absorption of food material; whereas, the third part is called as hindgut, responsible for excretion. Until recently, the digestion and absorption of lipids in insects have much neglected field of research, as indicated by review of insect lipid metabolism. Fats are the important dietary constituents in most of insects, and it must be assumed that, digestive lipases are on important erzymes (Turunen 1979, and Estela et.al 20010).

The neutral lipid values in male adults midgut was 61.86 while in female adult midgut it was 18.44 $\mathrm{mg} / \mathrm{gm}$. Wet weight of tissues. This finding suggested that the male midgut exhibited three fold more neutral lipid than the female midgut. The percentage of neutral lipids in midgut of male and female adults were 90.64 and 88.27 respectively. The NL:PL ratio in male and female midgut was 10:1 and 7:1 respectively. The above data indicated that the species investigated exhibit more neutral lipids than phospholipids. The diet is the probable source of neutral lipids in the midgut. Cmelik (1971) reported that the NL:PL ratio in termite queen midgut was $2: 1$. In the present investigation more neutral lipids than phospholipids, hence NL:PL ratio was differs than Cmelik's findings. The neutral lipids consisted monacylglycerol (MG), cholesterol (CHO), free fatty acids (FFA), diacylglycerol (DG), triacylglycerol (TG) and cholesterol ester (CE). TG consisted major component, MG \& DG in moderate concentration, whereas $\mathrm{CHO}, \mathrm{CE}$ and FFA in low concentration. The TG percentage of the neutral lipids in male midgut was 62.63 depleted to 32.81 in female midgut. This observation are agreed with Cmeliks finding (1971). On termite queen. He further stated that the midgut of insected were actively engaged in lipid digestion and absorption. In addition to that Reisser-Bollade (1976) reported that midgut also participate in converting TG into DG and FFA in P.americana.

The phospholipids values in male midgut was $6.392 \mathrm{mg} / \mathrm{gm}$. decreases to $2.450 \mathrm{mg} / \mathrm{gm}$. wet weight of tissues in female midgut. The male midgut exhibited three fold more phospholipids than female midgut. The phosphotidyl-choline (PC) and phosphatidyl-ethanolamine (PE) where major phospholipids constituents. Lysophophatidyl-choline (LPC), sphingomyelin (SPG), phosphotidyl inositol (PI), phosphatidyl serine (PS) and phosphatidic acid (PA) were minor constituents. This observation agreed with the observation of Cmelik (1971) on terminte queen. The percentage of PC and PE in relation to PL in male midgut was 39.07 and 37.6 enhanced to 49.02 and 38.00 in female midgut. This finding supports Cmelik (1971) on termite queen. 


\section{Acknowledgement}

Author thanks Dr. B. K. Pawar, Dept. of Zoology, Shivaji University, Kolhapur for his encouragements.

\section{References}

[1]. Abell, L.L., Levy, B.B., Brodie B.B., and Kendall, F.F. : A simplified method for the estimation of total cholesterol in serum and demonstration of its specificity., J. Biol. Chem. 195, 357-359 (1952).

[2]. Barwal, R.N. and Kalra, R.L. : Lipids lindane susceptible and resistant strains of T. castaneu., Indian J. Expt. Biol., 26, 228-234 (1988).

[3]. Cmelik, S.H.W., Composition of lipids from the guts of termite queens., J. Insect Physiol., 17, 1349-1358(1971).

[4]. Estela L. A.,Lilian E. C.,Zeina E.J., James E. P., Kozo T.,and Michael A. W., Lipid storage and mobilization in insects:Current status and future directions. Insect Biochemistry and molecular Biology.31,7-17 (2001)

[5]. Gloster, J. Flectar, R.F., Quantitative analysis of serum lipids with thin layer chromatography., clin. Chim. Acta., 13, 235-240 (1966).

[6]. Folch, J., Lees, M. and Sloane-Stanley A simple method for the isolation and purification of the total lipid from animal tissue. G.H. J.Biol. Chem 226 (1957) 497-509.

[7]. Hoffman, A.G.D. and Downer, R.G.H., : End product specificity of TG lipases from intestine fat body muscle and hemolymphy of the American cockroach, P. Americana., Lipids., 14, 893-899 (1979).

[8]. Hoffman, A.G.D., Alan, G.D, and Downer, R.G.H., : Synthesis of diglycerol by acetyltransferase from crop, midgut and fat body tissue of P.americana., Insect. Biochem., 9, 129-134 (1979).

[9]. Joshi, M. and Agarwal, H.C., : Cholesterol absorption in the cockroach P. americana., Entomon., 1, 93-100 (1976)

[10]. Joshi, M. and Agarwal, H.C., : Site of cholesterol absorption in some insects., J. Insect Physiol., 23, 403-404 (1977).

[11]. Lolage, G.R. and Patil S.P., : J. Maharashtra Agric Univ., 13, 23-25 (1978).

[12]. Male, K.B. and Storey, K.B., : Enzyme activities and isozyme composition of TG, DG, MG-lipases in P. americana, L. migratoria and P. adjuncta., Insect Biochem., 11, 423-427 (1981).

[13]. Marintti, G.V., : ed. "Lipid Chromatography Analysis". Vol I Marcel Dekker. Inc., New York. (1967).

[14]. Rimoldi, O.D., Peluffo, R.O., Gonzalez, S.M. and Brenner, R.R.,: Lipid digestion and transport in T. infestans., Comp. Biochem. Physiol, B. Comp. Biochem., 82, 187-190 (1985).

[15]. Skipski, V.P., Barclay, M., Reichman, E.S. and Good, J.J., : Biolchem, Biophys. Acta., 137, 80 (1967).

[16]. Turunen, S., : Digestion and absorption of lipids in insects., Comparative Biochem. Physiol., 63, 455-460 (1978).

[17]. Viogue, E, and Holaman, R.T., : Quantitative estimation of esters by thin layer chromatography., J.Am.oil. chemistis. Soc., 39, 68$66(1962)$

[18]. Wagner, H., Horhammer, L. and Wolfe, P., : Thin layer chromatography of phospholipids and glycolipids., Biochem., 2, 175-184 (1961).

Table No. 1 Alterations in total lipids ,neutral lipids and its components in male and female midgut of L.lepidophora.

\begin{tabular}{|c|c|c|}
\hline & Male midgut & Female midgut \\
\hline Total Lipids & $68.25 \pm 5.80$ & $20.89 \pm 1.04$ \\
\hline Neutral Lipids & $61.86 \pm 3.03$ & $18.44 \pm 0.92$ \\
\hline MG & $6.456 \pm 0.32$ & $5.280 \pm 0.26$ \\
\hline CHO & $1.968 \pm 0.04$ & $1.524 \pm 0.07$ \\
\hline FFA & $0.443 \pm 0.04$ & $0.313 \pm 0.01$ \\
\hline DG & $12.91 \pm 0.64$ & $3.310 \pm 0.16$ \\
\hline TG & $38.74 \pm 1.93$ & $6.051 \pm 6.051$ \\
\hline CE & $1.332 \pm 0.06$ & $1.960 \pm 0.09$ \\
\hline
\end{tabular}

The values for total lipids ,neutral lipids and its components are expressed as mg/gm. Wet weight of tissues.

Table No. 2 Alterations in phospholipids and its constituents in male and female midgut of L.lepidophora.

\begin{tabular}{|c|c|c|}
\hline & Male midgut & Female midgut \\
\hline Phospholipids & $6.392 \pm 0.30$ & $2.450 \pm 0.12$ \\
\hline PI & $8.231 \pm 0.43$ & $3.920 \pm 0.19$ \\
\hline LPC & $17.45 \pm 0.93$ & $1.980 \pm 0.09$ \\
\hline SPG & $19.23 \pm 0.99$ & $2.940 \pm 0.14$ \\
\hline PS & $9.312 \pm 0.43$ & $2.940 \pm 0.14$ \\
\hline PC & $99.90 \pm 5.10$ & $48.04 \pm 2.40$ \\
\hline PE & $96.23 \pm 5.92$ & $37.24 \pm 1.86$ \\
\hline PA & $5.328 \pm 0.31$ & $0.981 \pm 0.04$ \\
\hline
\end{tabular}

The values for phospholipids are expressed as $\mathrm{mg} / \mathrm{gm}$ wet weight of tossues; whereas, values of individual constituents are expressed in $\mu \mathrm{g}-\mathrm{P} / \mathrm{gm}$. Wet weight of tissues. 


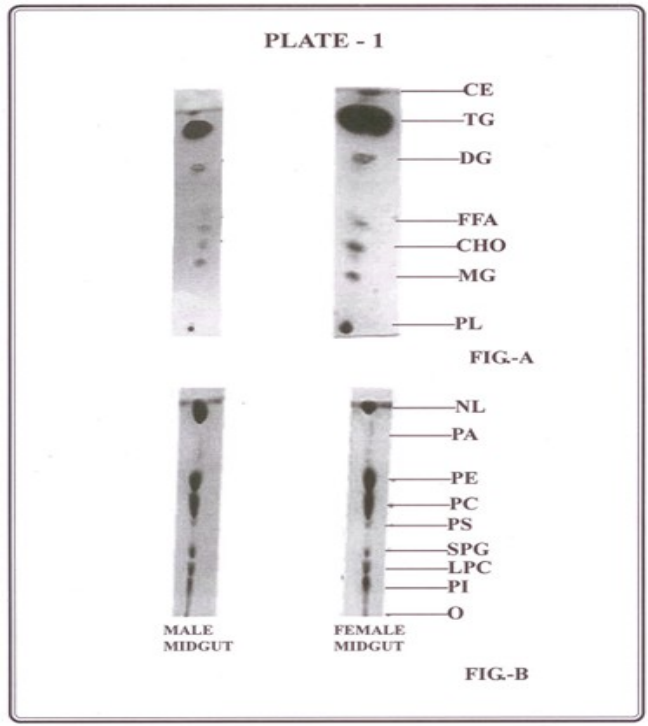

